\title{
Table of cases before the Court of Justice of the European Union (CJEU)
}

Case 30/59, De Gezamenlijke

Steenkolenmijnen in Limburg $v$.

High Authority of the European

Coal and Steel Community [1961]

E.C.R. 1 211, 212

Case 25/62, Plaumann v.

Commission [1963] E.C.R.

$95 \mathbf{3 8 0 ,}, \mathbf{3 8 3}$

Case 26/62, Van Gend en Loos

[1963] E.C.R. 1320

Case 6/64, Costa v. ENEL [1964]

E.C.R. 585 320, 350

Case 28/67, Molkerei-Zentrale

Westfalen v. Hauptzollamt

Paderborn [1968] E.C.R.

143291

Joined Cases 10 and 18/68,

Eridania and Others $v$.

Commission [1969] E.C.R.

$459 \mathbf{3 8 3}$

Case 47/69, France v. Commission [1970] E.C.R. 487 216, 292

Case 22/70, AETR [1971] E.C.R. 263320

Case 40/72, Schröeder v. Germany

[1973] E.C.R. $125 \quad 385$

Case 70/72, Commission v.

Germany [1973] E.C.R. 813 215

Case 120/73, Lorenz v. Germany [1973] E.C.R. 1471 24, 291, 351, 401

Case 173/73, Italy v. Commission

[1974] E.C.R. 709 191, 211, 213, 214
Case 36/74, Walrave and Koch v. Association Union Cycliste Internationale [1974] E.C.R. 1405291

Case 36/75, Rutili [1975] E.C.R. 1219320

Case 13/76, Donà v. Mantero [1976] E.C.R. 1333291

Case 74/76, Iannelli \& Volpi v. Meroni [1977] E.C.R. 557 291, 401

Case 78/76, Steinike und Weinlig v. Germany [1977] E.C.R. 595292

Case 82/77, van Tiggele [1978]

E.C.R. 25211

Case 106/77, Simmenthal [1978]

E.C.R. $629 \mathbf{3 2 0}$

Case 14/78, Denkavit v.

Commission [1978] E.C.R.

$2497 \mathbf{3 8 5}$

Case 91/78, Hansen v.

Hauptzollamt Flensberg [1979]

E.C.R. 935291

Case 73/79, Commission v. Italy

[1980] E.C.R. 1533291

Case 730/79, Philip Morris Holland $B V$ v. Commission [1980] E.C.R.

24, $\quad 267185,170,215,380,386$

Joined Cases 142 and 143/80,

Finanze dello Stato v. Essevi

and Salengo [1981] E.C.R.

1413291

Case 17/81, Pabst \& Richarz v.

Hauptzollamt Oldenburg [1982]

E.C.R. 1331291 
xvi Research handbook on European State aid law

Case 60/81, IBM v. Commission [1981] E.C.R. $2639 \quad 378$

Case 249/81, Commission v. Ireland [1982] E.C.R. 4005291

Joined Cases 296 and 318/82, Leeuwarder Papierwarenfabriek $B V$ v. Commission [1985] E.C.R. 80985

Case 323/82, Intermills $v$. Commission [1984] E.C.R. 3809383

Case 69/83, Luxembourg v. Court of Auditors [1984] E.C.R. 2447385

Case 17/84, Commission v. Ireland [1985] E.C.R. 2375291

Case 18/84, Commission v. France [1985] E.C.R. 1339291

Case 42/84, Remia BV and Verenigde Bedrijven Nutricia v. Commission [1985] E.C.R. $2545 \mathbf{3 8 5}$

Case 103/84, Commission v. Italy [1986] E.C.R. 1759291

Joined Cases 142 and 156/84, BAT and Reynolds v. Commission [1987] E.C.R. $4487 \mathbf{3 8 5}$

Case 169/84, Cofaz and others v. Commission [1986] E.C.R. 391383

Case 234/84, Belgium v. Commission [1986] E.C.R. 2263122

Joined Cases 67, 68 and 70/85, $R$ Van der Kooy v. Commission [1988] E.C.R. 219 104, 381

Case 187/85, Fediol v. Commission [1988] E.C.R. $4155 \mathbf{4 2 3}$

Case 282/85, DEFI v. Commission [1986] E.C.R. 2469381

Case 310/85, Deufil v. Commission [1987] E.C.R. $901 \quad \mathbf{3 8 0 ,} 386$
Case 131/86, United Kingdom v. Council [1988] E.C.R. 905 380

Case C-102/87, France v. Commission [1988] E.C.R. 4067 212, 215

Case C-142/87, Belgium v. Commission [1990] E.C.R. I-959 104, 352

Case C-301/87, France v. Commission (Boussac) [1990] E.C.R. I-307 122, 350, 352, 386, 401

Case C-21/88, Du Pont de Nemours Italiana [1990] E.C.R. I-889 291

Case C-175/88, Biehl v. Administration des contributions du Luxembourg [1990] E.C.R. I-1779 291

Case C-303/88, Italy v. Commission [1993] E.C.R. I-1433 271

Case C-261/89, Italy v. Commission [1991] E.C.R. I-4437 123

Case C-305/89, Italy v. Commission [1991] E.C.R. I-1603 122

Case C-309/89, Codorniu v. Council [1994] E.C.R. I-1853 381

Case C-10/90, Masgio Bundesknappschaft [1991] E.C.R. I-1119 291

Case C-41/90, Höfner and Elser [1991] E.C.R. I-1979 271

Joined Cases C-78 to C-83/90, Compagnie Commerciale de l'Ouest [1992] E.C.R. I-1847 291

Case C-179/90, Merci convenzionali porto di Genova $\operatorname{SpA}$ v.

Siderurgica Gabrielli SpA [1991] E.C.R. I-5889 273 
Table of cases before the CJEU xvii

Case C-303/90, France v. Commission [1991] E.C.R. I-5315 380

Case C-312/90, Spain v. Commission [1992] E.C.R. I-4117 351, 381

Case C-313/90, CIRFS and Others v. Commission [1991] E.C.R. I-2557 380, 388

Case C-354/90, Fédération Nationale de Commerce Extérieur des Produits Alimentaires and Others v. France [1991] E.C.R. I-5505 401

Case C-17/91, Lornoy [1992] E.C.R. I-6523 291

Case C-47/91, Italy v. Commission [1992] E.C.R. I-4145 351

Cases C-72 and C-73/91, Firma Sloman Neptun Schiffahrts $A G v$. Seebetriebsrat Bodo Ziesemer der Sloman Neptun Schiffahrts $A G$ [1993] E.C.R. I-887 211, 212

Case C-198/91, Cookv. Commission [1993] E.C.R. I-2487 377, 380-382, 384

Case C-225/91, Matrav. Commission [1993] E.C.R. I-3203 378, 381, 382, 384

Case C-325/91, France v. Commission [1993] E.C.R. I-3283 380

Case C-17/92, FEDICINE v. Spain [1993] E.C.R. I-2239 291

Case C-188/92, TWD Textilwerke Deggendorf v. Germany [1994] E.C.R. I-833 377, 401

Case C-387/92, Banco de Crédito Industrial $S A$, now Banco Exterior de España $S A v$. Ayuntamiento de Valencia [1994] E.C.R. I-877 211, 291
Case C-56/93, Belgium v. Commission [1996] E.C.R. I-723 191, 385, 386

Case C-39/94, SFEI v. Others [1996] E.C.R. I-3547 350

Case C-84/94, United Kingdom v. Council [1996] E.C.R. I-5755 386

Case C-233/94, Germany v. Parliament and Council [1997] ECR I-2405 386

Case C-241/94, France v. Commission (Kimberley Clark) [1996] E.C.R. I-4551 191, 213, 214

Case C-311/94, Ijssel-Vliet v. Minister van Economische Zaken [1996] E.C.R. I-5023 388

Case C-24/95, Alcan [1997] E.C.R. I-1491 402

Joined Cases C-192 to 218/95, Société Comateb and others [1997] E.C.R. I-165 216

Case C-241/95, Accrington Beef [1996] E.C.R. I-6699 402

Joined Cases C-248 and 249/95, SAM Schiffahrt and Stapf [1997] E.C.R. I-4475 386

Case C-367/95P, Commission v. Sytraval and Brink's France [1998] E.C.R. I-1719 378, 381, 382, 384, 387

Case C-147/96, Netherlands v. Commission [2000] E.C.R. I-4723 378

Case C-157/96, National Farmers' Union and Others [1998] E.C.R. I-2211 386

Case C-180/96, UKv v. Commission [1998] E.C.R. I-2265 385

Case C-213/96, Outokumpu Oy [1998] E.C.R. I-1777 292 
xviii Research handbook on European State aid law

Case C-342/96, Spain v. Commission [1999] E.C.R. I-2459 191

Case C-75/97, Belgium v. Commission (Maribel) [1999] E.C.R. I-3671 191, 213, 214 Case C-200/97, Ecotrade Srlv. Altiforni e Ferriere di Servola $\operatorname{SpA}(A F S)$ [1998] E.C.R. I-7907 213, 401

Case C-295/97, Piaggio [1999] E.C.R. I-3735 352

Joined Cases C-15/98 and C-105/99, Italy and Sardinia Lines v. Commission [2000] E.C.R. I-8855 381, 384

Case C-83/98P, France v. Ladbroke Racing and Commission [2000] E.C.R. I-3271 385, 386

Case C-99/98, Austria v. Commission [2001] E.C.R. I-1101 351

Case C-106/98P, Comité d'entreprise de la Societé française de production and others v. Commission [2000] E.C.R. I-3659 384

Case C-156/98, Germany v. Commission [2000] E.C.R. I-6857 190, 212, 271, 291

Case C-332/98, France v. Commission (CELF) [2000] E.C.R. I-4833 292, 423

Case C-351/98, Spain v. Commission [2002] E.C.R. I-8031 123, 380

Case C-352/98P, Bergaderm and Goupil v. Commission [2000] E.C.R. I-5291 385

Case C-379/98, PreussenElektra $A G$ and Schleswag $A G$ [2001] E.C.R. I-2099 41, 212, 272
Case C-390/98, Banks [2001] E.C.R. I-6117 213, 216, 402

Case C-480/98, Spain v. Commission [2000] E.C.R. I-8717 215

Case C-143/99, Adria-Wien Pipeline and others [2001] E.C.R. I-8365 191, 213, 214, 272, 275

Case C 194/99P, Thyssen Stahl v. Commission [2003] E.C.R. I-10821 $\mathbf{3 8 5}$

Case C-208/99, Portugalv. Commission [2001] E.C.R.

\section{I-9183 380}

Case C-234/99, Niels Nygård v. Svineafgiftsfonden [2002] E.C.R. I-3657 292

Case C-239/99, Nachi Europe GmbH v. Hauptzollamt Krefeld [2001] E.C.R. I-1197 402

Case C-382/99, Netherlands v. Commission [2002] E.C.R. I-5163 123, 212, 216, 387

Case C-453/99, Courage v. Crehan [2001] ECR I-6297 402

Case C-475/99, Glockner [2001] E.C.R. I-8089 324

Case C-482/99, France v. Commission [2002] E.C.R. I-4387 104, 272

Case C-53/00, Ferring $S A$ and $A C O S S$ [2001] E.C.R. I-9067 213, 216

Case C-113/00, Spain v. Commission [2002] E.C.R. I-7601 291

Case C-114/00, Spain v. Commission [2002] E.C.R. I-7657 291 
Table of cases before the CJEU xix

Joined Cases C 204, 205, 211, 213, 217 and 219/00P, Aalborg Portland and Others v. Commission [2004] E.C.R. I-123 385

Case C-209/00, Commission v. Germany [2002] E.C.R. I-11695 401

Case C-242/00, Germany v. Commission [2002] E.C.R. I-5603 380

Case C-280/00, Altmark Trans and Regierungspräsidium Magdeburg [2003] E.C.R. I-7747 273, 292, 320, 321

Case C-501/00, Spain v. Commission [2004] E.C.R. I-6717 190, 191

Joined Cases C-34 to 38/01, Enirisorse v. Ministero delle Finanze [2003] E.C.R. I-14243 292, 321

Case C-126/01, Ministère de l'Économie, des Finances et de l'Industrie v. GEMO SA [2003] E.C.R. I-13769 272, 273, 321 Joined Cases C-261 and 262/01, Van Calster with Cleeren and Slachthuis [2003] E.C.R. I-12249 216, 292, 352, 402

Case C-264/01, AOK Bundesverband et al. [2004] E.C.R. I-2493 324

Case C-308/01, GIL Insurance Ltd and others [2004] E.C.R. I-4777 191, 192, 213, 214, 272

Case C-383/01, De Danske Bilimportører [2003] E.C.R. I-6065 292

Case C-174/02, Streekgewest Westelijk Noord-Brabant $v$. Staatssecretaris van Financiën [2005] E.C.R. I-85 216, 292
Case C-175/02, F.J. Pape v. Minister van Landbouw, Natuurbeheer en Visserij [2005] E.C.R. I-127 216

Joined Cases C-189, 202, 205 to 208 and 213/02 P, Dansk Rørindustri and others $v$. Commission [2005] E.C.R. I-5425 380, 387

Case C-78/03 P, Aktionsgemeinschaft Recht und Eigentum v. Commission [2005] E.C.R. I-10737 378, 380-384

Case C-88/03, Portugal v. Commission (Azores) [2006] E.C.R. I-7115 190, 213, 215

Joined Cases C-128 and 129/03, EM SpA, AEM Torino $S p A v$. Autorità per l'energia elettrica e per il gas and Others [2005] E.C.R. I-2861 216

Case C-172/03, Wolfgang Heiser v. Finanzamt Innsbruck [2005] E.C.R. I-1627 191, 212, 213 Joined Cases C-182 and 217/03, Belgium and Forum 187 v. Commission [2006] E.C.R. I-5479 212, 380, 381, 402

Case C-205/03P, FENIN v. Commission [2006] E.C.R. I-6295 324

Case C-276/03P, Scott v. Commission [2005] E.C.R. I-8437 379

Case C-446/03, Marks \& Spencer plc v. David Halsey [2005] E.C.R. I-10837 291

Case C-451/03, Servizi Ausiliari Dottori Commercialisti Srl v. Giuseppe Calafiori [2006] E.C.R. I-2941 321 
Case C-148/04, Unicredito Italiano SpA v. Agenzia delle Entrate, Ufficio Genova [2005] E.C.R. I-11137 191

Case C-196/04, Cadbury Schweppes and Cadbury Schweppes Overseas [2006] E.C.R. I-7995 291

C-222/04, Ministero dell'Economia e delle Finanze v. Cassa di Risparmio di Firenze SpA [2006] E.C.R. I-289 291, 402

Case C-237/04, Enirisorse v. Sotacarbo [2006] E.C.R. I-2843 192

Joined Cases C-266 to 270, 276 and 321 to $325 / 04$, Distribution Casino France SAS [2005] E.C.R. I-9481 216, 292

Case C-368/04, Transalpine Ölleitung in Österreich and Others [2006] E.C.R. I-9957 352, 401, 402

Joined Cases C-393/04 and C-41/05, Air Liquide Industries Belgium $S A$ v. Ville de Seraing and Province de Liège [2006] E.C.R. I-5293 61, 216, 271, 273, 335, 402

Case C-525/04 P, Spain v. Lenzing [2007] E.C.R. I-9947 380, 384, 386, 387

Case C-526/04, Laboratoires Boiron $S A$. [2006] E.C.R. I-7529 216

Joined Cases C-75 and 80/05P, Germany v. Kronofrance and Others [2008] E.C.R. I-6619 380-382, 384

Case C-260/05, Sniace v. Commission [2007] E.C.R. I-10005 383, 384

Case C-266/05P, Sison v. Council [2007] E.C.R. I-1233 386
Case C-326/05P, Industrias

Quimicas del Vallés v.

Commission [2007] E.C.R. I-6557 387

Case C-341/05, Laval un Partneri Ltd v. Svenska [2007] E.C.R. I-11767 335

Case C-438/05, International Transport Workers' Federation, Finnish Seamen's Union v. Viking Line ABP, OÜ Viking Line Eesti [2007] E.C.R. I-10779 335

Case C-39/06, Commission v. Germany [2008] E.C.R. I-93 352

Case C-199/06, CELF [2008] E.C.R. I-469 276, 350, 352, 401

Case C-206/06, Essent Netwerk Noord BV and others [2008] E.C.R. I-5497 272, 292

Case C-341/06P, Chronopost [2008] E.C.R. I-4777 322, 324

Case C-346/06, Dirk Rüffert v. Land Niedersachsen [2009] E.C.R. 1989335

Case C-419/06, Commission v. Greece [2008] E.C.R. I-27 352

Joined Cases C-428 to 434/06, Unión General de Trabajadores de La Rioja (UGT Rioja) [2008] E.C.R. I-6747 190, 215

Case C- 487/06P, British Aggregates v. Commission [2008] ECR I-10505 190-194, 272, 381, 382, 384

Case C-494/06P, Commission v. Italian Republic and Wam Spa [2009] E.C.R. I-3639 86

Joined Cases C-501, 513, 515 and 519/06P, GlaxoSmithKline Services v. Commission [2009] E.C.R. I-9291 385 
Table of cases before the CJEU $\mathrm{xxi}$

Case C-521/06 P, Athinaïki

Techniki v. Commission [2008]

E.C.R. I-5829 378, 379, 384

Case C-214/07, Commission

v. France [2008] E.C.R.

I-8357 352

Case C-222/07, UTECA [2009]

E.C.R. I-1407 291

Case C-290/07P, Commission v.

Scott, judgment of 2 September 2010 385, 388

Case C-300/07, Oymanns/AOK Rheinland [2009] E.C.R. I-4779 324

Case C-319/07P, $3 F v$. Commission [2009] E.C.R. I-5963 382, 383

Case C-369/07, Commission v. Hellenic Republic [2009] E.C.R. I-5703 352

Case C-384/07, Wienstrom [2008] E.C.R. I-10393 352, 401

Case C-431/07P, Bouygues and Bouygues Télécom v. Commission [2009] E.C.R. I-2665 190

Case C-504/07, Antrop and Others v. Carris and STCP [2009]

E.C.R. I-3867 322

Case C-520/07P, Commission v. MTU [2009] E.C.R. I-8555 352

Case C-567/07, Woningstichting Sint Sevatius [2009] E.C.R. I-9021 324
Case C-160/08, Commission v. Germany, Judgment of 29 April 2010324

Case C 169/08, Presidente del Consiglio dei Ministriv. Regione Sardegna [2009] E.C.R. I-10821 213, 291

Case C-265/08, Federutility, judgment of 20 April $2010 \quad 323$

Case C-279/08P, Commission v. Netherlands (2008) OJ C 223/30 191

Case C-362/08P, Internationaler Hilfsfonds v. Commission, Judgment of 26 January 2010378

Case C-543/08, Commission v. Portugal, Judgment of 11 November $2010 \quad 321$

Case C-1/09, CELF v. SIDE, Judgment of 11 March 2010401

Case C-140/09, Fallimento Traghetti del Mediterraneo $S p A$ v. Presidenza del Consiglio dei Ministri, Judgment of 10 June 2010321

Case C-124/10, Commission appeal against judgment in Case T-156/04 Électricité de France (EDF) (2010) OJ C $161 / 16$ 104, 273, 322 